\title{
ON THE LEBEDEV-MILIN INEQUALITY ${ }^{1}$
}

\author{
MiSHAEL, ZEDEK
}

\begin{abstract}
A generalization is given of an inequality of Lebedev-Milin involving the coefficients of a power series $f$ and those of $\exp (f)$. It is proved that the exponential function can be replaced by certain functions belonging to the farrily of functions whose Taylor expansions at the origin have nonnegative coefficients.
\end{abstract}

In 1965 Lebedev and Milin [3] introduced a simple inequality which proved extremely useful in geometric function theory. We mention only three results where their inequality was used: (a) Milin [4] proved for $f(z)=z+\sum_{n=2}^{\infty} a_{n} z^{n} \in S$ (S) the class of normalized univalent functions in $|z|<1)$ that $\left|a_{n}\right|<(1.243) n$. (b) Il'ina [2] proved for $f \in S$ that ||$a_{n+1} \mid-$ $\left|a_{n}\right|<4.26$. (c) Aharonov [1] and Nehari [5] proved for $F(z)=$ $\sum_{n=;}^{\infty} b_{n} z^{n} \in$ B.E. (B.E. the Bieberbach-Eilenberg class of functions $F(z)$ reguiar in $|z|<1$ and $F(z) F(\zeta) \neq 1$ for any $|z|,|\zeta|<1)$ that $\sum_{n=1}^{\infty}\left|b_{n}\right|^{2} \leqq 1$.

We will give here a generalization of their inequality using the same method of proof as in [3].

THEOREM. Suppose that $g(t)=1+\sum_{k=1}^{\infty} p_{k} t^{k}, 0 \leqq p_{k}$, converges for $|t|<R, 0<R \leqq \infty$, and that the transformed inverse function $g^{-1}(1 /(1-s))=$ $\sum_{k=i}^{\infty} q_{k} s^{k}$ has positive coefficients. Let $\left\{A_{k}\right\}_{k=1}^{\infty}$ be an arbitrary sequence of complex numbers satisfying $\sum_{k=1}^{\infty}\left|A_{k}\right|^{2} \mid q_{k}<R$. Then from

follows

$$
g\left(\sum_{k=1}^{\infty} A_{k} z^{k}\right)=\sum_{k=0}^{\infty} D_{k} z^{k}
$$

$$
\sum_{k=0}^{\infty}\left|D_{\dot{k}}\right|^{2} \leqq g\left(\sum_{k=1}^{\infty}\left|A_{k}\right|^{2} / q_{k}\right),
$$

with equality if and only if $A_{k}=q_{k} \rho^{k} \eta^{k}$, where $0 \leqq \rho<[1-1 / g(R)]^{1 / 2}$ and $|\eta|=1$.

Received by the editors October 8, 1971.

AMS 1969 subject classifications. Primary 3009; Secondary 2670, 3042, 3043.

Key wora's and phrases. Lebedev-Milin inequalities, coefficient inequalities, univalent furictions, Bieberbach-Eilerberg functions.

: This research was supported by the National Science Foundation Grant GP-28970 to the University of Maryland.

(c) American Mathematicul Society 1972 
Proof. Denoting $a_{k}=\left|A_{k}\right| / q_{k}$ we set

and

$$
g\left(\sum_{k=1}^{\infty} a_{k} q_{k} z^{k}\right)=\sum_{k=0}^{\infty} B_{k} z^{k}
$$

$$
g\left(\sum_{k=1}^{\infty} a_{k}^{2} q_{k} z^{k}\right)=\sum_{k=0}^{\infty} C_{k} z^{k}
$$

Since $0 \leqq p_{k}$, we have $\left|D_{k}\right| \leqq B_{k}$. Comparing coefficients,

$$
\begin{array}{ll}
B_{0}=1, & B_{2}=p_{1} q_{2} a_{2}+p_{2} q_{1}^{2} a_{1}^{2}, \\
B_{1}=p_{1} q_{1} a_{1}, & B_{3}=p_{1} q_{3} a_{3}+2 p_{2} q_{1} q_{2} a_{1} a_{2}+p_{3} q_{1}^{3} a_{1}^{3},
\end{array}
$$

and in general $B_{k}=\sum_{\mu} \alpha_{\mu, k} U_{\mu, k}$ where $\alpha_{\mu, k}$ are nonnegative coefficients depending on $p_{i}$ and $q_{i}(i=1, \cdots, k)$ and $U_{\mu, k}$ are products of powers of $a_{i}(i=1, \cdots, k)$ of degree $k$. Clearly, $C_{k}=\sum_{\mu} \alpha_{\mu, k} U_{\mu, k}^{2}$. Also, if we set all $a_{k}=1$, we get

$$
g\left(\sum_{k=1}^{\infty} q_{k} z^{k}\right)=g\left(g^{-1}(1 /(1-z))\right)=\sum_{k=0}^{\infty} z^{k}=\sum_{k=0}^{\infty} \sum_{\mu} \alpha_{\mu, k} z^{k}
$$

and hence $\sum_{\mu} \alpha_{\mu, k}=1$ for $k=1,2, \cdots$.

By Cauchy's inequality

$$
B_{k}^{2}=\left\{\sum_{\mu}\left(\alpha_{\mu, k}\right)^{1 / 2}\left(\left(\alpha_{\mu, k}\right)^{1 / 2} U_{\mu, k}\right)\right\}^{2} \leqq \sum_{\mu} \alpha_{\mu, k} \cdot \sum_{\mu} \alpha_{\mu, k} U_{\mu, k}^{2}=C_{k}
$$

and so $\sum_{k=0}^{\infty}\left|D_{k}\right|^{2} \leqq \sum_{k=0}^{\infty} B_{k}^{2} \leqq \sum_{k=0}^{\infty} C_{k}=g\left(\sum_{k=1}^{\infty}\left|A_{k}\right|^{2} / q_{k}\right)$ which proves (2).

The conditions for equality are easily verified by observing that we must have $U_{\mu, k}=c_{k}$ and recursively $U_{\mu, k}=\rho^{k}\left(\rho=c_{1}\right)$. Hence $\left|A_{k}\right|=q_{k} a_{k}=q_{k} \rho^{k}$ and finally $\left|D_{k}\right|=B_{k}$ implies $A_{k}=q_{k} \rho^{k} \eta^{k}$ where $|\eta|=1$.

Note that the special case $p_{k}=1 / k$ ! and $q_{k}=1 / k$ gives the classic LebedevMilin inequality:

$$
\exp \sum_{k=1}^{\infty} A_{k} z^{k}=\sum_{k=0}^{\infty} D_{k} z^{k}
$$

implies

$$
\sum_{k=0}^{\infty}\left|D_{k}\right|^{2} \leqq \exp \sum_{k=1}^{\infty} k\left|A_{k}\right|^{2}
$$

\section{REFERENCES}

1. Dov Aharonov, On Bieberbach Eilenberg functions, Bull. Amer. Math. Soc. 76 (1970), 101-104. MR 41 \#1994.

2. L. P. Il'ina, The relative growth of nearby coefficients of schlicht functions, Mat. Zametki 4 (1968), 715-722. (Russian) MR 39 \#1644. 
3. N. A. Lebedev and I. M. Milin, An inequality, Vestnik Leningrad. Univ. 20 (1965), no. 19, 157-158. (Russian) MR 32 \#4248.

4. I. M. Milin, Estimaition of coefficients of univalent functions, Dokl. Akad. Nauk SSSR 160 (1965), 769-771 = Soviet Math. Dokl. 6 (1965), 196-198. MR 30 \#3206.

5. Z. Nehari, On the coefficients of Bieberbach-Eilenberg functions, J. Analyse Math. 23 (1970), 297-303.

Department of Mathematics, University of Maryland, College Park, MaryLAND 20742 